\title{
Blind separation of BPSK sources with residual carriers
}

\author{
Alle-Jan van der Veen
}

\begin{abstract}
Blind separation of instantaneous mixtures of binary sources with equal carrier frequencies has been studied before. For independent sources, it may be reasonable to assume that their carrier frequencies are not exactly identical, so that a residual carrier is present after demodulation to baseband. We show how this can be used to separate the sources. If the receiving antenna array is centro-symmetric and there is no multipath, then the performance of the algorithm can be significantly improved.
\end{abstract}

Keywords: blind source separation, constant modulus algorithm, residual carrier, blind beamforming

\section{INTRODUCTION}

Several techniques have been developed to estimate and separate linear superpositions of signals impinging onto an antenna array. They can broadly be characterized as (1) those that use properties of the channel, such as a parametrized multipath model and a known or structured antenna array, and (2) those that use properties of the signals. Some of the properties used in the latter category are training (known source symbols), constant modulus and finite alphabet of the source symbols, cyclo-stationarity, and statistical independence. For each of these, several methods are available to estimate the mixing matrix. Typical schemes are based on cost-function optimization using gradient-search or iterative techniques. Such methods are very much dependent on accurate initial points. There are also noniterative (algebraic) methods that are based on weak assumptions such as statistical independence of the sources (e.g., JADE [1], ICA [2], CANDECOMP [3]). These are very powerful in their generality but may require many samples before the higher-order statistics converge.

For short data bursts, we are interested in algebraic algorithms in which the mixing matrix is found as the best-fitting solution to a set of algebraic equations which pose conditions on every individual sample. For constant-modulus signals, a successful algorithm is the Analytic Constant Modulus Algorithm (ACMA) [4], which solves an overdetermined set of quadratic equations. The algorithm has been specialized to separate superpositions of binary $\{ \pm 1\}$ or $\{0,1\}$ signals [5]. In the present paper, we consider binary sources and explore ways to account for the fact that carrier frequencies are not always $100 \%$ identical.

In the case of digital signals from independent sources, it is reasonable to assume that the carriers are slightly different. E.g., suppose that the sources are modulated to $900 \mathrm{MHz}$ and that the carriers are the same up to some 5-6 orders of magnitude. After demodulation to baseband using the nominal carrier, each of the sources will have a residual carrier of up to $\pm 5 \mathrm{kHz}$, say. If the sources have a bandwidth of $20 \mathrm{kHz}$, then we can expect a phase

Delft Univ. of Technology, Dept. Elec. Eng./DIMES, 2628 CD Delft, The Netherlands.

${ }^{0}$ This paper was prepared in part while the author was on leave at ENST Paris, Dept. SIG, Paris, France, sponsored by ENST, and at Stanford University, Dept. ISL, Stanford, CA.

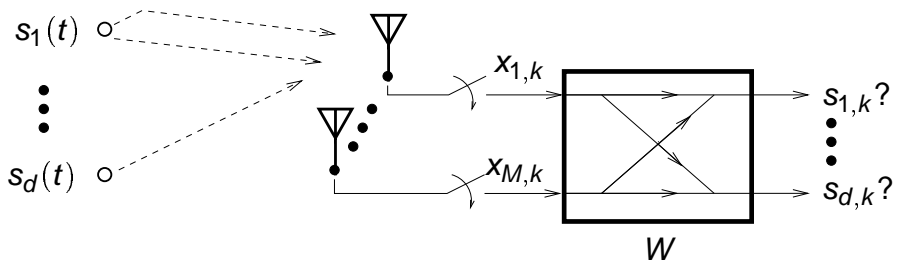

Fig. 1. Beamforming scenario

roll in the order of $\pm 90^{\circ}$ per symbol. Hence, the BPSK model $s_{k} \in\{ \pm 1\}$ is too naive in this case. Since the constant modulus property is still valid, we can revert to the ACMA to separate the sources. However, we can also try to separate them based on these small differences in residual carriers.

Modern-day communication systems use a common reference signal, so that the residual carrier is typically much smaller, reportedly around $500 \mathrm{~Hz}$ or less. As shown in the simulations, the residual carrier methods proposed here already work once the phase shift between the first and last symbol in the data batch is more than $\pm 180^{\circ}$. For sources with a bandwidth of $20 \mathrm{kHz}$ and a difference in carriers of $500 \mathrm{~Hz}$, this amounts to a data batch of 20 samples. We can envision systems where co-channel users are deliberately shifted by such small amounts, in order to facilitate separation. This can be regarded as a special instance of separation by "coding-induced cyclo-stationarity", and such schemes have been proposed e.g., in [6-8]. ${ }^{1}$

\section{Notation}

Vectors are denoted by boldface, matrices by capitals. Overbar $\left(^{-}\right)$denotes complex conjugation, ${ }^{T}$ is the matrix transpose, * the matrix complex conjugate transpose, and $\dagger$ is the matrix pseudo-inverse (Moore-Penrose inverse). $I_{m}$ is the $m \times m$ identity matrix, $\mathbf{0}$ and $\mathbf{1}$ are vectors for which all entries are equal to 0 and 1 , respectively. $\otimes$ is the Kronecker product, $\diamond$ is the "KhatriRao" product, which is a column-wise Kronecker product:

$A \otimes B=\left[\begin{array}{ccc}a_{11} B & a_{12} B & \cdots \\ a_{21} B & a_{22} B & \cdots \\ \vdots & \vdots & \ddots\end{array}\right], \quad A \diamond B=\left[\begin{array}{llll}\mathbf{a}_{1} \otimes \mathbf{b}_{1} & \mathbf{a}_{2} \otimes \mathbf{b}_{2} & \cdots\end{array}\right]$

Two notable properties are: $\operatorname{vec}(A B C)=\left(C^{T} \otimes A\right) \operatorname{vec}(B), \operatorname{vec}\left(\mathbf{a b}^{*}\right)=$ $\overline{\mathbf{b}} \otimes \mathbf{a}$, where the vec-operator indicates a stacking of the columns of a matrix into a vector. 


\section{DATA MODEL}

A source model for a unit-modulus BPSK source with some residual carrier is

$$
\mathbf{s}=\left[s_{k}\right] \in B P S K \quad \Leftrightarrow \quad s_{k} \in\left\{ \pm 1 \cdot \beta e^{j 2 \pi f k}\right\} \quad(k=1, \cdots, N) .
$$

Here, $\mathbf{s}$ is a row vector, $f$ is the unknown residual carrier frequency of the source and $\beta$ accounts for an initial phase $(|\beta|=1)$. We consider a scenario as depicted in figure 1 , where $M$ antennas receive linear superpositions of $d$ BPSK sources. Suppose we have collected a data block $X=\left[\mathbf{x}_{1} \cdots \mathbf{x}_{N}\right] \in \mathbb{C}^{M \times N}$. If the multipath delay spread is small relative to the sampling period, then $X$ is described by the standard model

$$
X=A S=\mathbf{a}_{1} \mathbf{s}_{1}+\cdots+\mathbf{a}_{d} \mathbf{s}_{d},
$$

where all $\mathbf{s}_{i} \in B P S K$. We assume that $d \leq M$, and that $A$ and $S$ have full rank $d$. The objective is to retrieve all (nontrivial) BPSK signals present in $X$, i.e. to find a collection of $d$ complex beamforming vectors $\mathbf{w}$ that lead to linearly independent BPSK signals

$$
\mathbf{s}=\mathbf{w}^{*} X
$$

of the form (1). A solution $\left\{\mathbf{w}_{1}, \cdots, \mathbf{w}_{d}\right\}$ is collected in a beamforming matrix $W=\left[\mathbf{w}_{1} \cdots \mathbf{w}_{d}\right]$. Since $\mathbf{s}$ has a sign ambiguity, we can recover $f$ from $\mathbf{s}$ only up to aliasing: $\hat{f} \in[-.25, .25\rangle$. Aliasing does not preclude demodulation.

To avoid nonuniqueness of $\mathbf{w}$ in case $d<M$ ( $X$ is rank deficient and arbitrary vectors from the left null space of $X$ could be added), we first replace $X$ by any full rank $d$ matrix $V=\left[\mathbf{v}_{1} \cdots \mathbf{v}_{N}\right]$ that has the same row span, for example as obtained from a singular value decomposition (SVD) of $X$ :

$$
X=U \Sigma V
$$

in which $U \in \mathbb{C}^{M \times d}$ and $V \in \mathbb{C}^{d \times N}$ are isometric, and $\Sigma \in \mathbb{R}^{d \times d}$ is diagonal. Note that $V=\Sigma^{-1} U^{*} X$, so that $\Sigma^{-1} U^{*}$ can be viewed as a pre-beamformer. ${ }^{2}$ After this step we look for all $d$ linearly independent vectors $\mathbf{t}$ such that

$$
\mathbf{t}^{*} V=\mathbf{s} \in B P S K \text {. }
$$

Since $A$ and $S$ are assumed to have full rank $d$, this equation has at least $d$ solutions $\left\{\mathbf{t}_{1}, \cdots, \mathbf{t}_{d}\right\}$ which together form the columns of a $d \times d$ matrix $T$. If a sufficient number of samples are taken, then it is known for the constant modulus case that generically there are precisely $d$ solutions (unique up to arbitrary initial phase) [4], and by extension, this applies to the BPSK decomposition as well. With $T$ in hand, the beamforming matrix $W$ on the original data is given by $W=U \Sigma^{-1} T$.

\section{SEPARATION USING CONSTANT MODULUS}

\section{Principle}

A first property that we can use to separate BPSK sources with residual carriers is their constant modulus:

$$
\left|s_{k}\right|=1 \quad \Leftrightarrow \quad s_{k} s_{k}^{*}=1 \quad(k=1, \cdots, N) .
$$

\footnotetext{
${ }^{1}$ Main differences are that we do not consider equalization, and that we treat the frequency offsets as unknown parameters.

${ }^{2}$ This step is also used to eliminate some of the additive noise. In this paper, we will not analyze noise effects in detail.
}

This leads to the ACMA [4] for the separation of constant modulus (CM) sources, which is summarized below for future reference. Substituting $s_{k}=\mathbf{t}^{*} \mathbf{v}_{k}$, we obtain

$$
\mathbf{t}^{*} \mathbf{v}_{k} \mathbf{v}_{k}^{*} \mathbf{t}=1 \quad \Leftrightarrow \quad\left(\overline{\mathbf{v}}_{k} \otimes \mathbf{v}_{k}\right)^{*}(\overline{\mathbf{t}} \otimes \mathbf{t})=1 \quad(k=1, \cdots, N)
$$

which can be compactly written as

$$
P \mathbf{y}=\mathbf{1}, \quad \text { where } \quad P:=(\bar{V} \diamond V)^{*}, \quad \mathbf{y}:=\overline{\mathbf{t}} \otimes \mathbf{t} .
$$

(Here, ' $\otimes$ ' is the Kronecker product and ' $\diamond$ ' denotes a columnwise Kronecker product.) Matrix $P$ has size $N \times d^{2}$, and we assume $N \geq d^{2}$. To solve for $\mathbf{t}$, we have to solve an overdetermined linear system in $\mathbf{y}$, where $\mathbf{y}$ is subject to a quadratic constraint, $\mathbf{y}=\overline{\mathbf{t}} \otimes \mathbf{t}$.

Any solution of the linear system in (2) can be written as

$$
\mathbf{y}=\alpha_{1} \mathbf{y}_{1}+\alpha_{2} \mathbf{y}_{2}+\cdots+\alpha_{\delta} \mathbf{y}_{\delta} \quad\left(\alpha_{1}=1\right)
$$

where $\mathbf{y}_{1}$ is a particular solution of the system, the other $\mathbf{y}_{k}$ are a basis of the null space of $P$, and $\delta-1$ is the dimension of this space. A convenient way to find the basis goes via a $Q R$ factorization of $\left[\begin{array}{ll}\mathbf{1} & P\end{array}\right]$ :

$$
Q^{*}\left[\begin{array}{ll}
\mathbf{1} & P
\end{array}\right]=\left[\begin{array}{cc}
r_{11} & \mathbf{r}_{12}^{T} \\
\mathbf{0} & \hat{P}
\end{array}\right] .
$$

(Matrix $\hat{P}$ has size $N-1 \times d^{2}$ but can be truncated to $d^{2} \times d^{2}$.) This replaces $P \mathbf{y}=\mathbf{1}$ by $\hat{P} \mathbf{y}=\mathbf{0}$, plus a nonessential scaling condition represented by the first row. Thus, $\left\{\mathbf{y}_{1}, \cdots, \mathbf{y}_{\delta}\right\}$ is a basis for the null space of $\hat{P}$ and can be found by an SVD of $\hat{P}$.

An important result is that, for sufficiently independent conditions, $\delta=d$ once $N>d^{2}-d$ [4]. The remaining problem is to find out which linear combinations of the $\left\{\mathbf{y}_{k}\right\}$ lead to a vector $\mathbf{y}$ that can be written as $\mathbf{y}=\overline{\mathbf{t}} \otimes \mathbf{t}$. The latter problem is conveniently rephrased by working with a matrix $Y=\mathbf{t t}^{*}$. For any matrix, we can form a vector by simply stacking its columns, and conversely, we can "unvec" vectors into matrices. A notable property is that $\mathbf{t t}^{*} \leftrightarrow \overline{\mathbf{t}} \otimes \mathbf{t}$. Thus, applying the unvec operation to every $\mathbf{y}_{k}$ in (3) then leads to the equivalent

$$
\mathbf{t t}^{*}=\alpha_{1} Y_{1}+\alpha_{2} Y_{2}+\cdots+\alpha_{\delta} Y_{\delta}
$$

Hence, the problem is to form linear combinations of known matrices $Y_{k}$ such that the result is rank-1 hermitian, so that it has a factorization as $\mathbf{t t}^{*}$. In the present case, there are $\delta=d$ matrices, and we are looking for all $d$ solutions $\mathbf{t}_{k}, k=1, \cdots, d$ to the problem: we are in fact trying to rewrite the given arbitrary "matrix basis" $\left\{Y_{k}\right\}$ as a rank-one matrix basis $\left\{\mathbf{t}_{k} \mathbf{t}_{k}^{*}\right\}$. The $\mathbf{t}_{k}$ are the columns of the beamforming matrix $T$.

The lucky situation that we have $\delta=d$ means that we can turn the argument around: we can write each matrix $Y_{k}$ as some linear combination of the rank-one basis:

$$
Y_{k}=\lambda_{k 1} \mathbf{t}_{1} \mathbf{t}_{1}^{*}+\lambda_{k 2} \mathbf{t}_{2} \mathbf{t}_{2}^{*}+\cdots+\lambda_{k d} \mathbf{t}_{d} \mathbf{t}_{d}^{*}, \quad(k=1, \cdots, d) .
$$

After collecting the coefficients into diagonal matrices, $\Lambda_{k}:=$ $\operatorname{diag}\left[\lambda_{k 1}, \cdots, \lambda_{k d}\right]$, it follows that the $Y_{k}$ satisfy the equations

$$
\begin{aligned}
Y_{1} & =T \Lambda_{1} T^{*} \\
& \vdots \\
Y_{d} & =T \Lambda_{d} T^{*}
\end{aligned}
$$


where all $\Lambda_{k}$ 's are diagonal. Thus, the matrices $\left\{Y_{k}\right\}$ are similar by congruence to diagonal matrices, and can be jointly diagonalized. This is essentially a generalized eigenvalue problem. Several algorithms are available, e.g., based on Jacobi iterations [1, $3,4,9-12]$. Since we usually have a good starting point from the eigenvalue problem of a pair of matrices, ${ }^{3}$ such iterations usually converge extremely fast, in two or three iterations, be it to a local optimum. After finding $T$, the beamformer on the original data is given by $W=U \Sigma^{-1} T$.

\section{Details}

A detail in the above algorithm is that we can use hermitian symmetry properties to map $\mathbf{y}=\overline{\mathbf{t}} \otimes \mathbf{t}$ to a real vector $\mathbf{y}^{\prime}:$ there is a data independent unitary matrix $J_{c}$ with a simple structure such that $J_{c} \mathbf{y}^{\prime}=\mathbf{y}$ with $\mathbf{y}^{\prime}$ real. This reduces the number of variables in $\mathbf{y}$ to $d^{2}$ real parameters. Note that $P \mathbf{y}=\mathbf{1} \Leftrightarrow\left(P J_{c}\right) \mathbf{y}^{\prime}=\mathbf{1}$. Since the rows of $P$ have a similar structure as $\mathbf{y}$, it is seen that $P^{\prime}=P J_{c}$ is also real. Thus, $P^{\prime} \mathbf{y}^{\prime}=\mathbf{1}$ can be solved in the real domain, the basis matrices $Y_{k}$ are complex hermitian by construction, so that the $\Lambda_{k}$ in (5) are real-valued.

In retrospect, it is essential in the above algorithm that $\delta=d$ : the null space dimension of $\hat{P}$ is equal to the number of sources. In general, the fact that there are $d$ solutions to the beamforming problem allows only to derive that $\delta \geq d$. A (weak) argument says that, for "sufficient excitation" by the sources and $A$ full rank, we can expect $\delta=d$, since random matrices have full rank with probability 1 . In our case, we have much more structured sources than in the CM case. The only thing we can prove easily is that $(i)$ the null space dimension of $\hat{P}$ is equal to that of $\bar{S} \diamond S$ and is independent of the actual $A$-matrix, since $P^{*}=$ $\bar{V} \diamond V=\left(T^{-1} \otimes T^{-*}\right)(\bar{S} \diamond S)$ with $T$ invertible, $(i i)$ the null space dimension of $\bar{S} \diamond S$ is too high if two sources have the same residual carrier frequencies $f_{i}$, leading to failure of the algorithm, (iii) for $d=2$, this is essentially the only exception. For $d>2$, the analysis becomes exceedingly complex.

There are important other applications where the $Y$-basis is larger than the rank-1 basis. This then leads to significant complications that have not yet been well studied.

\section{SEPARATION USING RESIDUAL CARRIERS}

\section{Outline}

The above constant modulus property does not use the more pertinent structure that is available for BPSK sources with residual carriers, cf. equation (1). If instead of $s_{k} s_{k}^{*}$ we look at $s_{k}^{2}$, we find

$$
s_{k}^{2}=\beta^{2} \phi^{k}, \quad \phi=e^{j 4 \pi f}
$$

Similar as before, substitute $s_{k}=\mathbf{t}^{*} \mathbf{v}_{k}$. In terms of Kronecker products, we can rewrite the equation as $\left[\mathbf{v}_{k} \otimes \mathbf{v}_{k}\right]^{T}(\overline{\mathbf{t}} \otimes \overline{\mathbf{t}})=\beta^{2} \phi^{k}$, or after complex conjugation, $\left[\mathbf{v}_{k} \otimes \mathbf{v}_{k}\right]^{*}(\mathbf{t} \otimes \mathbf{t})=\bar{\beta}^{2} \bar{\phi}^{k}$. If we col-

${ }^{3}$ This is the case if, for the selected pair $(i, j), Y_{i}$ is invertible and the eigenvalues $\Lambda_{j} \Lambda_{i}^{-1}$ of $Y_{j} Y_{i}^{-1}$ are distinct. lect the row vectors $\left[\mathbf{v}_{k} \otimes \mathbf{v}_{k}\right]^{*}$ in a matrix $P$ as before, we obtain $P \mathbf{y}=\bar{\beta}^{2}\left[\begin{array}{c}1 \\ \bar{\phi} \\ \vdots \\ \bar{\phi}^{N-1}\end{array}\right], \quad$ where $P:=(V \diamond V)^{*}, \quad \mathbf{y}:=\mathbf{t} \otimes \mathbf{t}, \quad|\phi|=1$.

Apart from a somewhat different definition of $P$ and $\mathbf{y}$, the main difference with the $\mathrm{CM}$ problem is the fact that right-hand side generated by $\phi$ is unknown. However, $\phi$ can readily be estimated using shift-invariance ideas from harmonic analysis, leading to an ESPRIT-type algorithm [13]. Let

$$
J^{(1)}:=\left[\begin{array}{ll}
I_{N-1} & \mathbf{0}
\end{array}\right], \quad J^{(2)}:=\left[\begin{array}{ll}
\mathbf{0} & I_{N-1}
\end{array}\right],
$$

then $P^{(1)}:=J^{(1)} P$ and $P^{(2)}:=J^{(2)} P$ are two shifts of $P$, and satisfy

$$
P^{(1)} \mathbf{y}=\bar{\beta}^{2}\left[\begin{array}{c}
1 \\
\bar{\phi} \\
\vdots \\
\bar{\phi}^{N-2}
\end{array}\right], \quad P^{(2)} \mathbf{y}=\bar{\beta}^{2}\left[\begin{array}{c}
\bar{\phi} \\
\bar{\phi}^{2} \\
\vdots \\
\bar{\phi}^{N-1}
\end{array}\right]
$$

so that

$$
P^{(1)} \mathbf{y}=\phi P^{(2)} \mathbf{y} .
$$

Hence, $\phi$ is a generalized eigenvalue of the matrix pencil $\left(P^{(1)}, P^{(2)}\right)$, and $\mathbf{y}$ is its corresponding eigenvector. We expect $d$ solutions that satisfy the equation. If the eigenvalues $\left\{\phi_{i}\right\}_{i=1}^{d}$ are distinct, the corresponding eigenvectors $\left\{\mathbf{y}_{i}\right\}_{i=1}^{d}$ are unique up to scaling, and can directly be factored as $\mathbf{y}_{i}=\mathbf{t}_{i} \otimes \mathbf{t}_{i} \cdot{ }^{4}$ As before, the $\mathbf{t}_{i}$ form the columns of the matrix $T$, and the beamforming matrix $W$ on the original data is given by $W=U \Sigma^{-1} T$. The $\phi_{i}$ allow to estimate the residual carriers, up to aliasing, since $\phi_{i}=\exp \left(j 4 \pi f_{i}\right)$. (Recall that the aliasing is inherent in the problem definition and does not preclude demodulation.)

\section{Details}

The current definition of $\mathbf{y}$ as $\mathbf{y}=\mathbf{t} \otimes \mathbf{t}$ has repeated entries: it can be regarded as a vectorization of the symmetric matrix $\mathbf{t t}^{T}$, which has only $\frac{1}{2} d(d+1)$ distinct complex variables. The repeated entries in $\mathbf{y}$ should be removed. Let $\mathbf{y}^{\prime}$ be the reducedsize vector with unique entries, then there is a data independent reconstruction matrix $J_{s}: d^{2} \times \frac{1}{2} d(d+1)$ with a simple structure such that $J_{s} \mathbf{y}^{\prime}=\mathbf{y}$. Note that $P \mathbf{y}=\left(P J_{s}\right) \mathbf{y}^{\prime}$. Thus if we let $P^{\prime}=P J_{s}$, then we can solve the reduced-size problem

$$
P^{\prime(1)} \mathbf{y}^{\prime}=\phi P^{\prime(2)} \mathbf{y}^{\prime},
$$

where $P^{(1)}, P^{\prime(2)} \in \mathbb{C}^{(N-1) \times \frac{1}{2} d(d+1)}$.

If $N>\frac{1}{2} d(d+1)$, then the matrices $P^{\prime(1)}$ and $P^{\prime(2)}$ are rectangular (tall), and the eigenvalue problem is known as a singular pencil problem. Such problems require special attention in cases where one of the matrices is singular: this should be avoided. Similarly as before, $V=T^{-*} S$ and $P^{*}=V \diamond V=\left(T^{-*} \otimes T^{-*}\right)(S \diamond$ $S)$, so that the rank of $P^{\prime}$ is only dependent on the sources and not on the particular $A$-matrix (assuming it is full rank). Again, $P^{\prime}$ is

\footnotetext{
${ }^{4}$ This factorization is easily obtained by going to the matrix domain: $Y_{i}=$ $\operatorname{vec}^{-1}\left(\mathbf{y}_{i}\right)=\mathbf{t}_{i} \mathbf{t}_{i}^{T}$. An SVD can be used to verify that it is rank 1.
} 
singular if two sources have the same frequencies (up to aliasing); for $d=2$, this is the only defective case. The general case again defies analysis, but generically, one expects that $P^{\prime}$ has full rank as long as frequencies are different (up to aliasing).

With $P^{\prime}$ full rank and rectangular (tall), there are several ways to solve $P^{\prime(1)} \mathbf{y}^{\prime}=\phi P^{\prime(2)} \mathbf{y}^{\prime}$. We could look at the eigenvalue decomposition of $P^{\prime(2) \dagger} P^{\prime(1)}$, but this is not fully correct: it produces $\frac{1}{2} d(d+1)$ eigenvalues out of which we have to select $d$. (The spurious extra eigenvectors do not satisfy (9).) The correct way to solve the pencil is to reduce the problem dimension by looking at the intersection of the column spans of $P^{\prime(1)}$ and $P^{\prime(2)}$, as follows.

Assume there are $d$ solutions to (9), and let $Y:=\left[\mathbf{y}_{1}^{\prime} \cdots \mathbf{y}_{d}^{\prime}\right]$ contain the $d$ eigenvectors. Then $P^{(1)} Y=P^{\prime(2)} Y \Phi$, where $\Phi=$ $\operatorname{diag}\left[\phi_{i}\right]$, i.e.,

$$
\left[\begin{array}{ll}
P^{\prime(1)} & P^{\prime(2)}
\end{array}\right]\left[\begin{array}{c}
Y \\
-Y \Phi
\end{array}\right]=0 .
$$

Thus, $\left[P^{\prime(1)} \quad P^{\prime(2)}\right]$ has (at least) a $d$-dimensional null space. Generically, the dimension will be precisely $d$ once the matrix is sufficiently tall, i.e., $N-1 \geq d(d+1)-d$, or $N>d^{2}$. Assuming this, a basis for the null space can be computed using an SVD, which then produces matrices $F$ and $G$ of size $\frac{1}{2} d(d+1) \times d$ such that

$$
\left[\begin{array}{ll}
P^{(1)} & P^{\prime(2)}
\end{array}\right]\left[\begin{array}{c}
F \\
-G
\end{array}\right]=0 .
$$

Since the basis is determined only up to multiplication by a nonsingular $d \times d$ matrix $M$, it follows that

$$
F=Y M, \quad G=Y \Phi M
$$

Consequently,

$$
F^{\dagger} G=M^{-1} \Phi M
$$

so that $\Phi$ and $M^{-1}$ can be found as the eigenvalues and eigenvectors, respectively, of $F^{\dagger} G$. Finally, the eigenvectors satisfying (9) are given by

$$
\left[\mathbf{y}_{1}^{\prime} \cdots \mathbf{y}_{d}^{\prime}\right]=F M^{-1} \text {. }
$$

From each $\mathbf{y}_{i}^{\prime}$, the corresponding $\mathbf{y}_{i}=J_{s} \mathbf{y}_{i}^{\prime}$ is recovered, and subsequently factored as $\mathbf{y}_{i}=\mathbf{t}_{i} \otimes \mathbf{t}_{i}$ to retrieve the beamforming matrix $T$.

The resulting algorithm is summarized in figure 2 . It requires $N>d^{2}$ samples and produces both beamforming vectors and demodulation frequencies. It fails when two eigenvalues are the same, i.e., two signals have precisely the same carrier frequency, since in that case $\left[P^{\prime(1)} P^{\prime(2)}\right]$ has a null space dimension which is too large, rendering the subsequent procedure invalid. There appears to be no easy solution to this problem.

\section{Discussion}

As can be guessed from comparing (6) to (2), the difference in accuracy to the constant modulus-based solution turns out to be only marginal. The main benefit in solving (6) is that it leads to a somewhat simpler and more standard eigenvalue problem in the final step, and the fact that estimates of residual carrier frequencies are available, which simplifies recovery of the source symbols after beamforming. The main step is the computation of an SVD of $\left[\begin{array}{ll}P^{\prime(1)} & P^{\prime(2)}\end{array}\right]$, a complex matrix of size $(N-1) \times d(d+1)$.

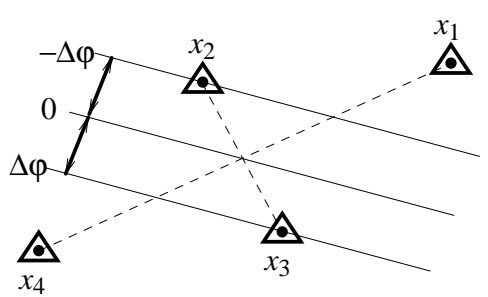

Fig. 3. A centro-symmetric array

In contrast, ACMA requires computation of an SVD of a realvalued matrix of comparable size, which takes only about $1 / 4$ of the amount of operations.

Although the source symbols are real-valued, this is not very well used by the procedure. In analogy to the situation without residual carrier (see [5]), we would hope for some form of realvalued processing, doubling of dimensions, and use of the fact that $|\phi|=1$. How to exploit the additional structure without making further assumptions remains an open question.

Another challenge at this point would be to combine both the constant modulus property $\left(\left|s_{k}\right|^{2}=1\right)$ and the residual carrier structure $\left(s_{k}^{2}=\beta^{2} \phi^{k}\right)$. At present, this is not possible because different parameter vectors are used: $\overline{\mathbf{t}} \otimes \mathbf{t}$ and $\mathbf{t} \otimes \mathbf{t}$, respectively.

\section{SeParation USing RESIDUAl CARRIERS AND A CENTRO-SYMMETRIC ARRAY}

\section{Outline}

The situation becomes more interesting if we can assume additional structure on $A$. Suppose that $(i)$ the array is centro-symmetric (see figure 3 ) and (ii) that there is no multipath. Such assumptions are often made for direction finding algorithms such as (unitary)ESPRIT [14]. The symmetry of the array carries over into a symmetry of the array manifold vectors, under assumption $(i i)$ equal to the columns of $A$ :

$$
\Pi \bar{A}=A, \quad \Pi=\left[\begin{array}{lll}
\mathbf{0} & & 1 \\
& . & \\
1 & & \mathbf{0}
\end{array}\right],
$$

where we have placed the zero phase reference in the center of the array. It follows that

$$
X=A S \quad \Rightarrow \quad \Pi \bar{X}=A \bar{S}
$$

so that

$$
\begin{array}{cc}
X \diamond \Pi \bar{X} & =(A \otimes A)(S \diamond \bar{S}) \\
X \diamond X & =(A \otimes A)(S \diamond S) .
\end{array}
$$

The factor $A \otimes A$ is the same in both expressions. Thus, due to the centro-symmetric property we can now try to find vectors of the form $\mathbf{y}=\mathbf{w} \otimes \mathbf{w}$ that reconstruct rows of $S \diamond \bar{S}$ and $S \diamond S$ of the form $\left[\begin{array}{llll}1 & 1 & 1 & \cdots\end{array}\right]$ and $\beta^{2}\left[\begin{array}{llll}1 & \phi & \phi^{2} & \cdots\end{array}\right]$, respectively. This gives twice as many conditions on the same parameter vector as we had before.

Again, $X$ is rank deficient when $d<M$, which leads to a nonuniqueness in $\mathbf{w}$ that has to be avoided. Since $\left[\begin{array}{ll}X & \Pi \bar{X}\end{array}\right]=A\left[\begin{array}{ll}S & \bar{S}\end{array}\right]$ has rank $d$, a suitable preprocessing step is to compute the SVD

$$
\left[\begin{array}{ll}
X & \Pi \bar{X}
\end{array}\right]=U \Sigma\left[\begin{array}{ll}
V_{1} & V_{2}
\end{array}\right]
$$


Given a matrix $X=A S+N$. An estimate of $S \in B P S K$ is obtained as follows:

1. Estimate $\operatorname{row}(X)$ :

a. Compute $\operatorname{SVD}(X): X=: U \Sigma V$

b. Estimate $d=\operatorname{rank}(X)$ from $\Sigma$ : the number of signals

c. Truncate $U, \Sigma, V$ to this rank.

2. Solve the pencil problem (9):

a. $P^{\prime}:=(V \diamond V)^{*} J_{s}$

b. $P^{\prime(1)}:=J^{(1)} P^{\prime}, P^{\prime(2)}:=J^{(2)} P^{\prime}$

c. Use SVD to find the $d$-dimensional null space $\left[\begin{array}{c}F \\ -G\end{array}\right]$ of $\left[\begin{array}{ll}P^{\prime(1)} & P^{\prime(2)}\end{array}\right]$

d. Eigenvalue decomposition $F^{\dagger} G=: M^{-1} \Phi M$

e. $Y:=J_{S} F M^{-1}$

3. Find the beamforming vectors and the source signals

a. Using SVD, factor each column $\mathbf{y}$ of $Y$ as $\mathbf{y}=: \mathbf{t} \otimes \mathbf{t}$

b. Using the resulting matrix $T$, set $W:=U \Sigma^{-1} T$

c. $S:=W^{*} X$

The entries of $\Phi$ specify the residual carrier frequencies.

Fig. 2. Algorithm Rescar, for separating BPSK sources with residual carriers.

in which $U \in \mathbb{C}^{M \times d}$ and $\left[V_{1} V_{2}\right] \in \mathbb{C}^{d \times 2 N}$ are isometric, and $\Sigma \in$ $\mathbb{R}^{d \times d}$ is diagonal. Singular vectors are unique up to unimodular scaling (if singular values are not repeated). Since $\left[\begin{array}{ll}\Pi \bar{X} & X\end{array}\right]=$ $U \Sigma\left[\begin{array}{ll}V_{2} & V_{1}\end{array}\right]$ but also $\left[\begin{array}{ll}\Pi \bar{X} & X\end{array}\right]=\Pi \bar{U} \Sigma\left[\bar{V}_{1} \quad \bar{V}_{2}\right]$, we see that there is a unimodular scaling of the columns of $U$ and rows of [ [ $\left.\begin{array}{ll}V_{1} & V_{2}\end{array}\right]$ such that

$$
\Pi \bar{U}=U, \quad V_{1}=\bar{V}_{2}=: V
$$

Assume that we take the SVD in this way. Denote as before by $T$ the nonsingular $d \times d$ beamforming matrix such that $T^{*} V=S$, then $U \Sigma=A T^{*}$. It follows from $\Pi \bar{U}=U, \Pi A=\bar{A}$ that $T=\bar{T}$ so that $T$ is real-valued.

We can now set up conditions for constant modulus and for residual carriers using a single real-valued vector $\mathbf{y}=\mathbf{t} \otimes \mathbf{t}$, where $\mathbf{t}$ is one of the columns of $T$. Since $\mathbf{t}=\overline{\mathbf{t}}$, equations (2) and (6) can immediately be combined. Thus define

$$
P_{1}=(\bar{V} \diamond V)^{*}, \quad P_{2}=(V \diamond V)^{*}, \quad \mathbf{y}=\mathbf{t} \otimes \mathbf{t},
$$

then $\mathbf{y}$ satisfies

$$
P_{1} \mathbf{y}=\left[\begin{array}{c}
1 \\
1 \\
\vdots
\end{array}\right], \quad P_{2} \mathbf{y}=\bar{\beta}^{2}\left[\begin{array}{c}
1 \\
\phi \\
\vdots
\end{array}\right], \quad\left(\phi=e^{j 4 \pi f}\right)
$$

The shift invariance of the second condition leads to $P_{2}^{(1)} \mathbf{y}=$ $\phi P_{2}^{(2)} \mathbf{y}$. Since $\mathbf{y}$ is real and $|\phi|=1$, we can also set up a conjugated set of conditions of the form $\bar{P}_{2}^{(2)} \mathbf{y}=\phi \bar{P}_{2}^{(1)} \mathbf{y}$ which can be combined with the second condition into a single eigenvalue problem

$$
\left[\begin{array}{c}
P_{2}^{(1)} \\
\bar{P}_{2}^{(2)}
\end{array}\right] \mathbf{y}=\phi\left[\begin{array}{c}
P_{2}^{(2)} \\
\bar{P}_{2}^{(1)}
\end{array}\right] \mathbf{y}
$$

Finally, if we map the first condition $P_{1} \mathbf{y}=\mathbf{1}$ to $\hat{P}_{1} \mathbf{y}=\mathbf{0}$ as before (cf. (4)), then we can combine all conditions into a single eigenvalue problem

$$
\begin{aligned}
& {\left[\begin{array}{c}
\hat{P}_{1} \\
0 \\
P_{2}^{(1)} \\
\bar{P}_{2}^{(2)}
\end{array}\right] \mathbf{y}=\phi\left[\begin{array}{c}
0 \\
\hat{P}_{1} \\
P_{2}^{(2)} \\
\bar{P}_{2}^{(1)}
\end{array}\right] \mathbf{y}} \\
& \Leftrightarrow \quad P_{A} \mathbf{y}=\phi P_{B} \mathbf{y}, \quad \mathbf{y}=\mathbf{t} \otimes \mathbf{t} .
\end{aligned}
$$

Equation (12) now plays the role of (8). A solution of the eigenvalue problem produces $\left\{\phi_{i}\right\}$ and $\left\{\mathbf{y}_{i}\right\}$, from which the frequencies $f_{i}$ and beamforming vectors $\mathbf{t}_{i}$ can be obtained. The latter are collected in a matrix $T$, after which the beamforming matrix on the original data is given by $W=U \Sigma^{-1} T$.

\section{Details}

The matrix $[X \Pi \bar{X}]$ can be mapped to a real matrix: there are data independent unitary matrices $Q_{1}$ and $Q_{2}$ with simple structures such that $Q_{1}^{*}[X \Pi \bar{X}] Q_{2}$ is real-valued [14]. Thus, the computation of the SVD of $[X \Pi \bar{X}]$ can be carried out in the real domain and $T$ will be real-valued without further effort.

Since $\mathbf{t}$ is real, $\mathbf{y}=\mathbf{t} \otimes \mathbf{t}$ can be parametrized by a vector $\mathbf{y}^{\prime}$ consisting of $\frac{1}{2} d(d+1)$ real parameters. There is a reconstruction matrix $J_{s}$ such that $\mathbf{y}=J_{s} \mathbf{y}^{\prime}$. Moreover, $J_{s}$ is such that $P_{1}^{\prime}=P_{1} J_{s}$ is real-valued as we had before. The same holds for $\hat{P}_{1}^{\prime}$. The first condition is thus mapped into $\hat{P}_{1}^{\prime} \mathbf{y}^{\prime}=\mathbf{0}$ with $\hat{P}_{1}^{\prime}$ real.

It is also possible to map the second condition to real-valued matrices. Indeed, since $|\phi|=1$, a Cayley transformation provides a one-to-one mapping of $\phi$ to $\lambda=j \frac{1-\phi}{1+\phi}$ which is real. The corresponding eigenvalue problem (11) is mapped to (see appendix)

$$
\begin{aligned}
& {\left[\begin{array}{c}
-\left(P_{i}^{(2)}-P_{i}^{(1)}\right) \\
P_{r}^{(2)}-P_{r}^{(1)}
\end{array}\right] \mathbf{y}=\lambda\left[\begin{array}{c}
P_{r}^{(1)}+P_{r}^{(2)} \\
P_{i}^{(1)}+P_{i}^{(2)}
\end{array}\right] \mathbf{y},} \\
& P_{r}:=\operatorname{real}\left(P_{2}\right), \quad P_{i}:=\operatorname{imag}\left(P_{2}\right) .
\end{aligned}
$$

The same holds for the primed quantities after transformation by 
$J_{s}$. Thus, (12) is transformed into

$$
\begin{gathered}
{\left[\begin{array}{c}
\hat{P}_{1}^{\prime} \\
0 \\
P_{i}^{\prime(1)}-P_{i}^{(2)} \\
P_{r}^{\prime(2)}-P_{r}^{\prime(1)}
\end{array}\right] \mathbf{y}=\lambda\left[\begin{array}{c}
0 \\
\hat{P}_{1}^{\prime} \\
P_{r}^{(1)}+P_{r}^{\prime(2)} \\
P_{i}^{\prime(1)}+P_{i}^{\prime(2)}
\end{array}\right] \mathbf{y}} \\
\Leftrightarrow P_{A}^{\prime} \mathbf{y}^{\prime}=\lambda P_{B}^{\prime} \mathbf{y}^{\prime}
\end{gathered}
$$

where $P_{A}^{\prime}, P_{B}^{\prime} \in \mathbb{R}^{4(N-1) \times \frac{1}{2} d(d+1)}$. As before, the singular pencil problem is solved by looking for the null space of $\left[\begin{array}{ll}P_{A}^{\prime} & P_{B}^{\prime}\end{array}\right]$ and solving the reduced $d$-dimensional eigenvalue problem, cf. (10) and the subsequent equations.

\section{Discussion}

In summary, the additional assumptions that the array is centrosymmetric and that there is no multipath allows to $(i)$ find a more accurate subspace filter $U$, based on $2 N$ vectors, (ii) construct roughly 4 times as many conditions on $\mathbf{y}$, which consists of half as many real parameters. Thus, we expect a significant improvement in accuracy over the previous schemes. The computational complexity is determined by the SVD of $\left[\begin{array}{ll}P_{A}^{\prime} & P_{B}^{\prime}\end{array}\right]$, a real matrix of size $4(N-1) \times d(d+1)$. Because it is real, the complexity is the same as that of the previous residual carrier algorithm in section IV, or four times the complexity of the ACMA in section III, although some reduction in complexity is possible.

\section{Simulations}

The performance of the algorithms discussed above is illustrated by means of a few simulations. In the basic simulation setup, we took $d=3$ BPSK sources, located at angles $\left[-15^{\circ}, 0,15^{\circ}\right]$ and with normalized residual frequencies $[-0.15,0,0.15]$. The antenna array consisted of a uniform linear array of $M=4$ sensors, spaced at $\frac{\lambda}{2}$. (This array configuration was chosen to enable a comparison to the ESPRIT algorithm.) The sources have equal unit power, the array has unit gain, and complex i.i.d. white Gaussian noise is added with variance $\sigma^{2} . N=20$ samples are taken, at symbol-spaced sampling rate.

The algorithms that are tested are 'ACMA' (section III), 'ACMA (same, but using the centro-symmetric structure of the array by acting on $\left[\begin{array}{ll}X & \Pi \bar{X}\end{array}\right]$ ), 'Rescar' (section IV), 'Rescar+c' (section $\mathrm{V})$, and 'ESPRIT+c', which is Unitary-ESPRIT and acts on $[X \Pi \bar{X}]$ $[14] .5$

Three performance criteria are shown. The first is the maximal residual signal-to-interference ratio (SIR) at the output of the beamformer, which is obtained by inspection of $W^{*} A$, where $W$ is the estimated beamforming matrix. The second is the signal to interference plus noise ratio after beamforming (SINR), and the third is the bit error rate (BER) after beamforming and demodulation. In the latter case, estimates for the residual carriers for the ACMA and ESPRIT algorithms are obtained using a single shift invariance property for each recovered signal independently.

For reference, plots show in dotted lines the performance of the 'optimal' SINR-maximizing beamformer with known $A, \sigma$

${ }^{5}$ Although the ESPRIT algorithm is primarily known as a direction-finding algorithm, its final step is an eigenvalue decomposition of which the eigenvalues provide the usual direction estimates and the eigenvector matrix is the beamforming matrix $T$. (but estimated residual frequencies) defined by

$$
\begin{aligned}
& W_{\mathrm{opt}}=\left[\begin{array}{lll}
\mathbf{w}_{1, \mathrm{opt}} & \cdots & \mathbf{w}_{d, \mathrm{opt}}
\end{array}\right] \\
& \mathbf{w}_{i, \mathrm{opt}}=R_{n n, i}^{-1} \mathbf{a}_{i}, \quad R_{n n, i}=\sigma^{2} I_{M}+A A^{*}-\mathbf{a}_{i} \mathbf{a}_{i}^{*} .
\end{aligned}
$$

The frequencies are estimated after beamforming in the same way as for the ACMA and ESPRIT algorithm.

Figure 4(a) features the performances as function of SNR. It is seen that for the above-mentioned parameter settings, the performance of ACMA is similar to that of Rescar, whereas there is a $3 \mathrm{~dB}$ performance improvement if the centro-symmetry is used. Although Rescar+c uses 4 times more conditions, it is not much more accurate than ACMA+c, whose conditions are a subset. With respect to SINR, the performances are close to optimal. The performance of ESPRIT+c is significantly lower, but this is strongly dependent on the angle separation, as we will see later.

Figure $4(b)$ shows the performance as a function of $N$, the number of samples. The SIR-performance of both ACMA and Rescar-based algorithms saturate, because they are biased by the noise (just as $W_{\text {opt }}$ is biased). The bias is natural because conditions are put on the output of the beamformer, although another source of bias is due to the squaring of the data. The BER rates go up because the frequency estimates do not sufficiently improve for growing $N$. This suggests the use of a more advanced frequency estimator.

In figure $4(c)$, the frequency separation between the sources is varied: the residual frequencies are taken to be $\left[-\Delta_{f}, 0, \Delta_{f}\right]$. The minimum frequency separation that is needed for Rescar is 0.025 . For this value, the phase difference between the first and last sample is precisely $0.025 N=0.5$, or $180^{\circ}$. This result seems to generalize: it has been verified for other values of $N$ that the minimum frequency separation required is $\frac{1}{2 N}$. ACMA also needs some frequency separation, otherwise it will fail because the null space dimension of $\hat{P}$ becomes too large. Use of the centro-symmetric structure lowers the minimum in all cases. Note that when $\Delta_{f}=0.25$, two frequencies are the essentially the same due to aliasing, so that the algorithms fail in that case as well. The performance drop of Rescar $+c$ is felt in a wider frequency region. This is likely due to the mapping of $\phi$ to $\lambda$ : in this region two of the eigenvalues become very large and go to infinity.

In figure $4(d)$, we vary the angle separation between the sources. This shows that the relatively inferior performance of ESPRIT in the previous graphs is in fact much dependent on the separation in angles between the sources. The angle dependence of the other algorithms is due to the change in condition numbers of $A$; for separations larger than $30^{\circ}$, the conditioning is close to 1 and the performance flattens out. For well-separated sources, ESPRIT can improve in SIR performance even over the nonblind case (known $S$ and $\left\{f_{i}\right\}$ ), because it uses structure of the $A$ matrix. However, the possible improvement in SINR and hence in BER is only small.

\section{CONCLUSION}

We have proposed two new algorithms for the blind separation of nonconvolutive BPSK sources based on differences in residual carriers, and compared them to ACMA and ESPRIT. Both new algorithms inherently estimate the residual carriers. The second algorithm also exploits centro-symmetry of the array and 

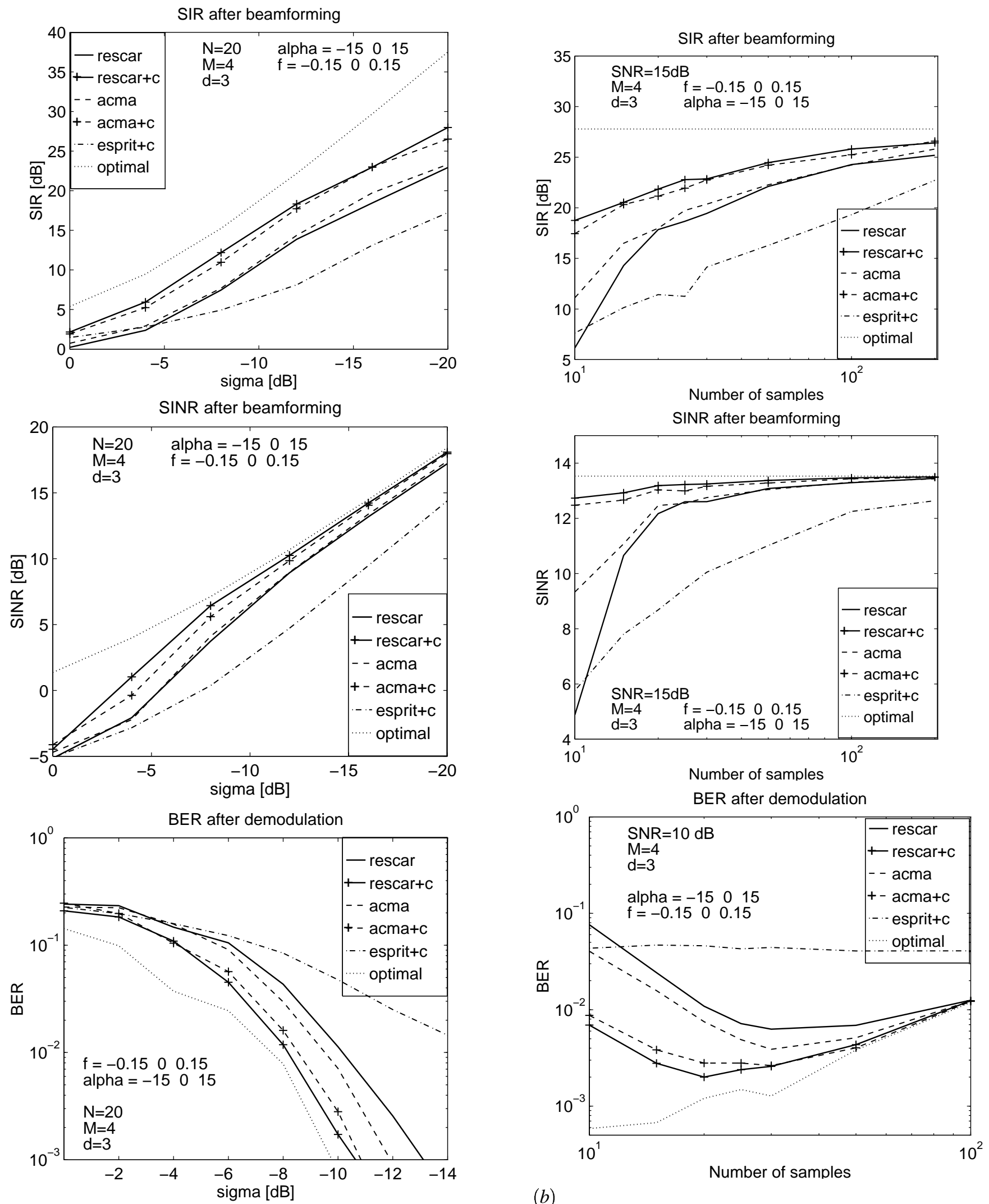

(a)

(b)

Fig. 4. Performance of blind separation algorithms. (a) Dependence of SIR, SINR and BER on noise power and $(b)$ on the number of samples. 

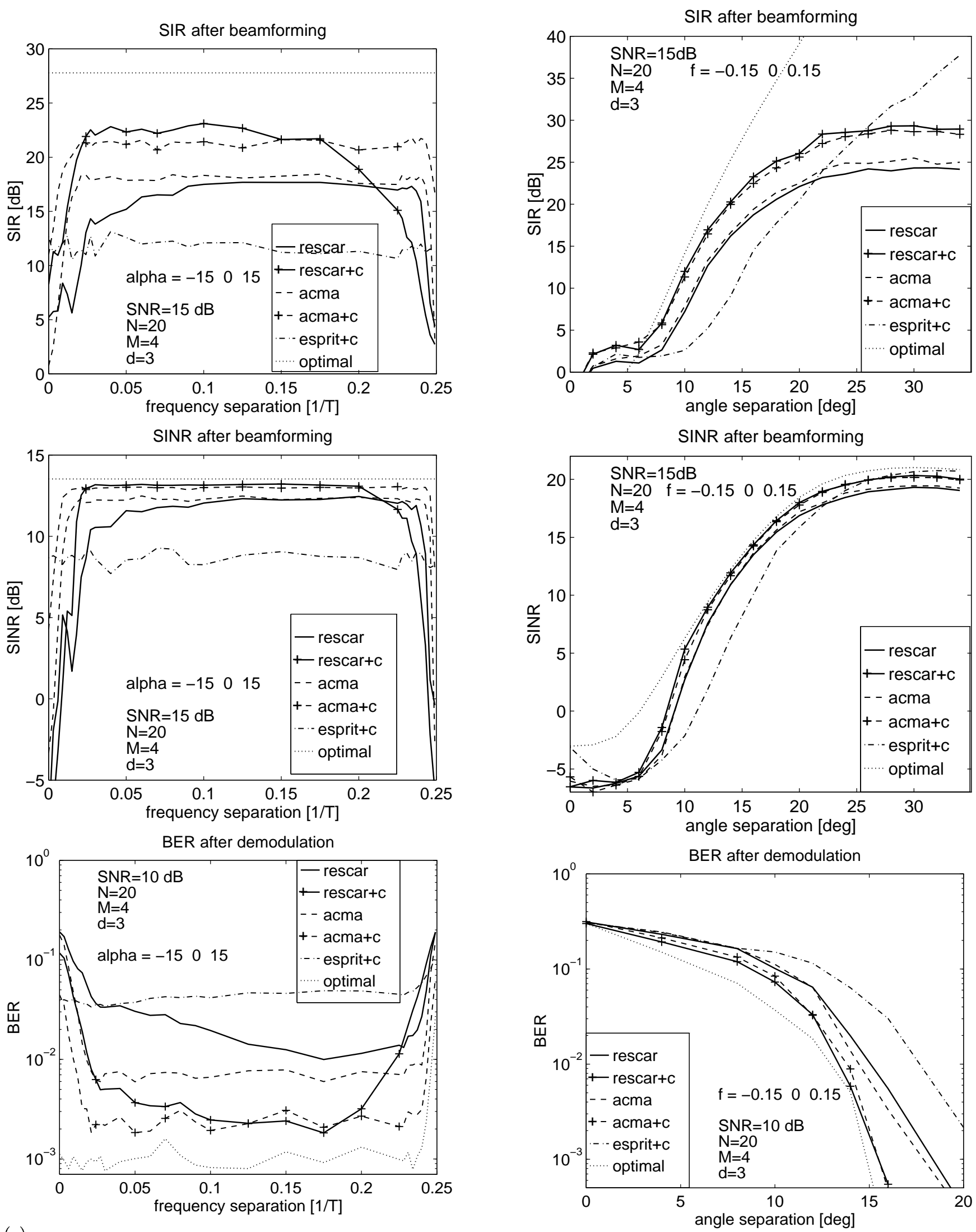

(c)

(d)

Fig. 4. [cont'd] (c) Dependence on frequency separation and $(d)$ on angle separation. 
absence of multipath, and combines with the constant-modulus property of the constellation. In most cases, the performance of the proposed algorithms is rather similar to ACMA, without or with using centro-symmetry respectively. To insure good performance, angle separation and frequency separation should be above certain minimum values that are inversely proportional to $M$ and $N$, respectively. The minimum frequency separation can also be lowered by subsampling the array outputs. Similarly, the minimum angle separation can be lowered by using arrays with longer baselines.

The new algorithms have a computational complexity that is 4 times that of ACMA. The main reason to prefer the new algorithms over ACMA would be that the final eigenvalue problem is standard and easier to implement, and that estimates of the residual carriers are directly available, facilitating subsequent demodulation. On the other hand, they are not applicable to modulations other than BPSK.

Among possible extensions of this work, we could mention

- Study convolutive channels, including the effect of ISI due to the pulse shape function, since without blind equalization this will introduce an apparent source with the same carrier frequency, for which the current algorithms fail,

- Systematic study of the impact of noise, and derivation of an optimal prewhitening.

\section{APPENDIX}

\section{CAYLEY TRANSFORMATION}

Consider the equation $A \mathbf{x}=\phi B \mathbf{x}$, with $\mathbf{x}$ real and $|\phi|=1$. Then

$$
\begin{aligned}
& (B-A) \mathbf{x}=(1-\phi) B \mathbf{x} \\
& (B+A) \mathbf{x}=(1+\phi) B \mathbf{x}
\end{aligned}
$$

so that

$$
j(B-A) \mathbf{x}=j \frac{1-\phi}{1+\phi}(B+A) \mathbf{x}
$$

Define $\lambda=j \frac{1-\phi}{1+\phi}$, then $\lambda$ is real and $\mathbf{x}$ is real, whence

$$
\left[\begin{array}{c}
-\operatorname{imag}(B-A) \\
\operatorname{real}(B-A)
\end{array}\right] \mathbf{x}=\lambda\left[\begin{array}{c}
\operatorname{real}(B+A) \\
\operatorname{imag}(B+A)
\end{array}\right] \mathbf{x} .
$$

This set of equations contains the conjugate equation $\bar{B} \mathbf{x}=\phi \bar{A} \mathbf{x}$ as well.

\section{REFERENCES}

[1] J.F. Cardoso and A. Souloumiac, "Blind beamforming for non-Gaussian signals," IEE Proc. F (Radar and Signal Processing), vol. 140, no. 6, pp. 362-370, Dec. 1993.

[2] P. Comon, "Independent component analysis, a new concept?," Signal Processing, vol. 36, no. 3, pp. 287-314, Apr. 1994.

[3] L. De Lathauwer, B. De Moor, and J. Vandewalle, "Independent component analysis based on higher-order statistics only," in Proc. IEEE SP Workshop on Stat. Signal Array Proc., Corfu, Greece, 1996, pp. 356-359.

[4] A.J. van der Veen and A. Paulraj, "An analytical constant modulus algorithm," IEEE Trans. Signal Processing, vol. 44, no. 5, pp. 1136-1155, May 1996.

[5] A.J. van der Veen, "Analytical method for blind binary signal separation," IEEE Trans. Signal Proc., vol. 45, no. 4, pp. 1078-1082, Apr. 1997.

[6] A. Chevreuil and Ph. Loubaton, "Blind second-order identification of FIR channels: forced cyclostationarity and structured subspace method," IEEE Signal Proc. Letters, vol. 4, no. 7, pp. 204-206, July 1997.

[7] M.K. Tsatsanis and G.B. Giannakis, "Coding induced cyclostationarity for blind channel equalization," in Proc. 29-th Conf. on Inform. Sciences and Systems, Baltimore (MA), 1995, pp. 685-690.
[8] B.G. Agee, S.V. Schell, and W.A. Gardner, "Spectral self-coherence restoral: A new approach to blind adaptive signal extraction using antenna arrays," Proc. IEEE, vol. 78, no. 4, pp. 753-767, Apr. 1990.

[9] P. Binding, "Simultaneous diagonalization of several Hermitian matrices," SIAM J. Matrix Anal. Appl., vol. 4, no. 11, 1990.

[10] M.T. Chu, "A continuous Jacobi-like approach to the simultaneous reduction of real matrices," Lin. Alg. Appl., vol. 147, pp. 75-96, 1991.

[11] A. Bunse-Gerstner, R. Byers, and V. Mehrmann, "Numerical methods for simultaneous diagonalization," SIAM J. Matrix Anal. Appl., vol. 4, pp. 927-949, 1993

[12] K. Abed-Meraim and Y. Hua, "A least-squares approach to joint Schur decomposition," in Proc. IEEE ICASSP, 1998, pp. 2541-2544.

[13] R. Roy and T. Kailath, "ESPRIT - Estimation of Signal Parameters via Rotational Invariance Techniques,” IEEE Trans. Acoust., Speech, Signal Proc., vol. 37, no. 7, pp. 984-995, July 1989.

[14] M. Haardt and J.A. Nossek, "Unitary ESPRIT: how to obtain increased estimation accuracy with a reduced computational burden," IEEE Trans. Signal Proc., vol. 43, no. 5, pp. 1232-1242, May 1995. 